\title{
Asymptomatic Multiple Lymphomatous Polyposis Identified during Staging Bidirectional Endoscopy of Mantle Cell Lymphoma
}

\author{
Sonja P. Dawsey ${ }^{a} \quad$ Jason A. Gregory ${ }^{b} \quad$ Alexander W. Brown ${ }^{c}$ \\ Frances J. Jones $^{d}$ \\ ${ }^{a}$ Department of Internal Medicine, Brooke Army Medical Center, San Antonio, Tex., USA; \\ ${ }^{b}$ Department of Pathology, Brooke Army Medical Center, San Antonio, Tex., USA; 'Division \\ of Hematology and Oncology, Brooke Army Medical Center, San Antonio, Tex., USA; \\ ${ }^{\mathrm{d}}$ Division of Gastroenterology, Brooke Army Medical Center, San Antonio, Tex., USA
}

\section{Keywords}

Multiple lymphomatous polyposis - Mantle cell lymphoma - Gastrointestinal neoplasms .

Endoscopy · Leptomeningeal

\begin{abstract}
Multiple lymphomatous polyposis (MLP) as an extranodal manifestation of mantle cell lymphoma $(\mathrm{MCL})$ in the gastrointestinal tract is rare and not often reported in the literature. We describe the case of a 63-year-old female with asymptomatic MLP found during staging bidirectional endoscopy of MCL. The patient presented only with dyspnea, but was found on physical exam to have diffuse lymphadenopathy, and subsequent positron emission tomography (PET) CT showed extensive lymph node adenopathy consistent with lymphoma. Excisional lymph node biopsy revealed high-risk MCL. Prior to therapy, staging bidirectional endoscopy was performed, which revealed duodenal bulb polyps and diffuse polyposis in the colon. Biopsies showed atypical lymphoid infiltrate identical to the initial excisional lymph node biopsy. The patient underwent aggressive induction therapy, chemotherapy and bone marrow transplantation. Four months later, repeat colonoscopy and biopsies showed normal mucosa, and repeat PET CT showed no evidence of systemic disease. Eight months later, the
\end{abstract}


patient began having symptoms consistent with cauda equina syndrome, and she was found to have leptomeningeal recurrence of MCL. In spite of other medical treatment, the patient's $\mathrm{MCL}$ progressed and she passed away 3 years after the initial presentation.

(c) 2016 The Author(s)

Published by S. Karger AG, Basel

\section{Introduction}

Mantle cell lymphoma (MCL) is a B-cell lymphoma that accounts for $6 \%$ of non-Hodgkin's lymphomas [1]. Extranodal spread of MCL is common, including to the bone marrow, blood, and the gastrointestinal (GI) tract. Multiple lymphomatous polyposis (MLP) is a rare manifestation of MCL affecting one or more GI tract segments. It was first described in 1961 as multiple sessile or pedunculated polyps [2]. Endoscopically, it can be unapparent, or it can result in a diffuse covering of small nodules or polyps [3]. Patients with MLP typically present with abdominal pain, diarrhea, and GI bleeding. We report an unusual case of a patient without GI symptoms who was found to have MLP affecting areas of the duodenum, colon, and rectum.

\section{Case Report}

A 63-year-old female presented with shortness of breath but denied B-symptoms (fevers, night sweats, weight loss). On physical exam, diffuse lymphadenopathy was discovered, a chest x-ray showed multiple enlarged perihilar lymph nodes, and a positron emission tomography (PET) CT showed extensive lymph node adenopathy above and below the diaphragm consistent with lymphoma. Excisional lymph node biopsy was performed, revealing an atypical lymphoid infiltrate that stained positive for CD5, CD20, and cyclin D1 and negative for CD23 and CD10. Proliferative index quantified by Ki-67 was $60 \%$ and lactate dehydrogenase (LDH) was $259 \mathrm{IU} / \mathrm{l}$. Fluorescence in situ hybridization of a bone marrow aspirate showed $\mathrm{t}(11 ; 14)(\mathrm{q} 13 ; \mathrm{q} 32)$, which is the classic genetic abnormality for MCL [4]. Due to her high proliferative index and increased LDH, the patient was classified as having high-risk disease by the Mantle Cell Lymphoma International Prognostic Index (MIPI) [5]. Prior to therapy, staging bidirectional endoscopy was performed. EGD revealed duodenal bulb polyps, and colonoscopy revealed diffuse polyposis (fig. 1). Biopsies of the duodenal bulb, ascending colon, and rectum showed involvement of the mucosa by an atypical lymphoid infiltrate with histologic morphology and immunohistochemical staining identical to that of the initial excisional lymph node biopsy.

The patient underwent aggressive induction therapy with rituximab + cyclophosphamide, vincristine, doxorubicin, and prednisone (R-CHOP), alternating with rituximab and high-dose cytarabine. Then she was given high-dose chemotherapy using carmustine, etoposide, cytarabine, and melphalan/cyclophosphamide (BEAM) with rituximab and autologous bone marrow transplantation. Four months later, repeat endoscopy with biopsies showed normal mucosa (fig. 2), and repeat PET CT showed no evidence of systemic disease. Eight months later, the patient began having pain in the coccyx region and symptoms consistent with cauda equina syndrome, and she was found to have a leptomeningeal relapse of MCL, with malignant cells identified in her cerebrospinal fluid. In spite of other medical treatment with high-dose steroids, rituximab, intrathecal and intraventricular methotrexate, allogenic bone marrow transplant, and radiation (L2-S3: 37.5 Gy; T7-S3: $30 \mathrm{~Gy}$ ), the patient's MCL 
progressed, and she also developed graft versus host disease. She was subsequently discharged to home hospice, and she passed away 3 years and 2 months after her initial diagnosis of MCL.

\section{Discussion}

MLP is a very rare disorder. When it occurs, it is most characteristically seen in patients with MCL [4]. MCL most commonly affects males (75-80\% of cases), and usually presents in the fifth or sixth decade of life [1]. The malignant cells originate from the mantle zone of lymphoid follicles and classically express CD5, CD20, and cyclin D1 [4]. They are also characterized by a $\mathrm{t}(11 ; 14)(\mathrm{q} 13 ; \mathrm{q} 32)$ translocation which causes a deregulation of the $\mathrm{Bcl}-1$ oncogene on chromosome 11, leading to overexpression of cyclin D1 [6]. To assess prognosis, the MIPI is used, which includes the factors age, sex, performance status, LDH level, WBC count, and the percentage of Ki-67-positive cells determined by immunohistochemistry [5]. The current patient had a Ki-67 of $60 \%$ and a MIPI of 7.3, both indicating high-risk disease with poor prognosis. MCL is an aggressive lymphoma and around 70\% of MLP patients are diagnosed at stage IV at presentation. The overall survival reported for all MCL patients is 3-4 years [7], and for stage IV MCL patients it is 29 months [5].

Although GI involvement of systemic MCL is relatively common, patients will typically present with GI complaints like diarrhea, abdominal pain, or rectal bleeding. These symptoms are similar to those of patients experiencing a flare of inflammatory bowel disease, and MLP should be kept in mind when evaluating individuals in the population of patients who present with these complaints [8]. In the current patient, no GI symptoms were reported, and only the staging bidirectional endoscopy found the MLP, which is quite uncommon. Although bidirectional endoscopy is considered essential in the workup of stage I and II MCL by the National Comprehensive Cancer Network, it is not routine in more advanced stages since it rarely leads to clinical management changes [9]. It is often used, however, to assess response to treatment.

The treatment for MLP is treating the underlying MCL. For many years, the standard of care for MCL was R-CHOP, which resulted in high response rates but a short duration of response before relapse, averaging 1.5 years [1]. Adding high-dose cytarabine to frontline therapy is a newer approach and can result in more durable responses, with progressionfree survival averaging 3 years [1]. Geisler et al. [10] of the Nordic Lymphoma Group also included high-dose BEAM and autologous stem cell rescue as consolidation therapy, resulting in an improved response duration of over 10 years. When risk was stratified, however, higher-risk patients had substantially reduced remission durations averaging only 2.8 years. The high-risk patient discussed here unfortunately suffered relapse only 8 months after intensive initial therapy.

Central nervous system (CNS) involvement by MCL is rare, probably occurring in $<10 \%$ of cases [11]. This spread of MCL to the CNS is usually a late event, and most commonly occurs in patients with high-risk disease. The current patient had high-risk disease that relapsed 8 months after an initial complete remission, and the presentation of her relapse was a cauda equina syndrome caused by leptomeningeal involvement. In spite of this unusual relapse, the patient still survived for 2 years after this recurrence.

In summary, we report an unusual patient with high-risk MCL who had asymptomatic MLP identified only at staging bidirectional endoscopy. She received aggressive immunotherapy, chemotherapy, and bone marrow transplantation, with a successful remission for 


\section{Case Reports in Oncology}

8 months, but eventually relapsed with CNS involvement, and died 3 years and 2 months after her initial diagnosis.

\section{Statement of Ethics}

The authors have no ethical conflicts to disclose.

\section{Disclosure Statement}

Open Access was funded by the Division of Hematology and Oncology of the Brooke Army Medical Center.

\section{References}

1 Skarbnik AP, Goy AH: Mantle cell lymphoma: state of the art. Clin Adv Hematol Oncol 2015;13:44-55.

-2 Cornes JS: Multiple lymphomatous polyposis of the gastrointestinal tract. Cancer 1961;14:249-257.

-3 Ruskone-Fourmestraux A, Audouin J: Primary gastrointestinal tract mantle cell lymphoma as multiple lymphomatous polyposis. Best Pract Res Clin Gastroenterol 2010;24:35-42.

-4 O'Malley DP, Goldstein NS, Banks PM: The recognition and classification of lymphoproliferative disorders of the gut. Hum Pathol 2014;45:899-916.

5 Hoster E, Dreyling M, Klapper W, et al: A new prognostic index (MIPI) for patients with advanced-stage mantle cell lymphoma. Blood 2008;111:558-565.

6 Bosman FT, Carneiro F, Hruban RH, et al: WHO Classification of Tumours of the Digestive System, ed 4. World Health Organization Classification of Tumours. Lyon, International Agency for Research on Cancer (IARC), 2010.

7 Weisenburger DD, Vose JM, Greiner TC, et al: Mantle cell lymphoma. A clinicopathologic study of 68 cases from the Nebraska Lymphoma Study Group. Am J Hematol 2000;64:190-196.

-8 Sciaudone G, Pellino G, Selvaggi F: Diagnostic pitfalls: cancerization in IBD versus mantle cell lymphoma presenting with multiple lymphomatous polyposis. Inflamm Bowel Dis 2011;17:E28-E30.

9 National Comprehensive Cancer Network: Non-Hodgkin's lymphoma: mantle cell lymphoma (version 2.2105). http://www.nccn.org/professionals/physician_gls/pdf/nhl.pdf (accessed September 18, 2015).

-10 Geisler CH, Kolstad A, Laurell A, et al: Nordic MCL2 trial update: six-year follow-up after intensive immunochemotherapy for untreated mantle cell lymphoma followed by BEAM or BEAC + autologous stem-cell support: still very long survival but late relapses do occur. Br J Haematol 2012;158:355-362.

11 Ferrer A, Bosch F, Villamor N, et al: Central nervous system involvement in mantle cell lymphoma. Ann Oncol 2008;19:135-141.

The views expressed herein are those of the authors and do not reflect the official policy or position of Brooke Army Medical Center, the U.S. Army Medical Department, the U.S. Army Office of the Surgeon General, the Department of the Army and Air Force, the Department of Defense, or the U.S. Government. 


\section{Case Reports in Oncology}

\begin{tabular}{l|l}
\hline Case Rep Oncol 2016;9:661-665 \\
\hline DOI: 10.1159/000450596 & $\begin{array}{l}\text { C 2 2016 The Author(s). Published by S. Karger AG, Basel } \\
\text { www.karger.com/cro }\end{array}$ \\
\hline
\end{tabular}

Dawsey et al.: Asymptomatic Multiple Lymphomatous Polyposis Identified during Staging Bidirectional Endoscopy of Mantle Cell Lymphoma
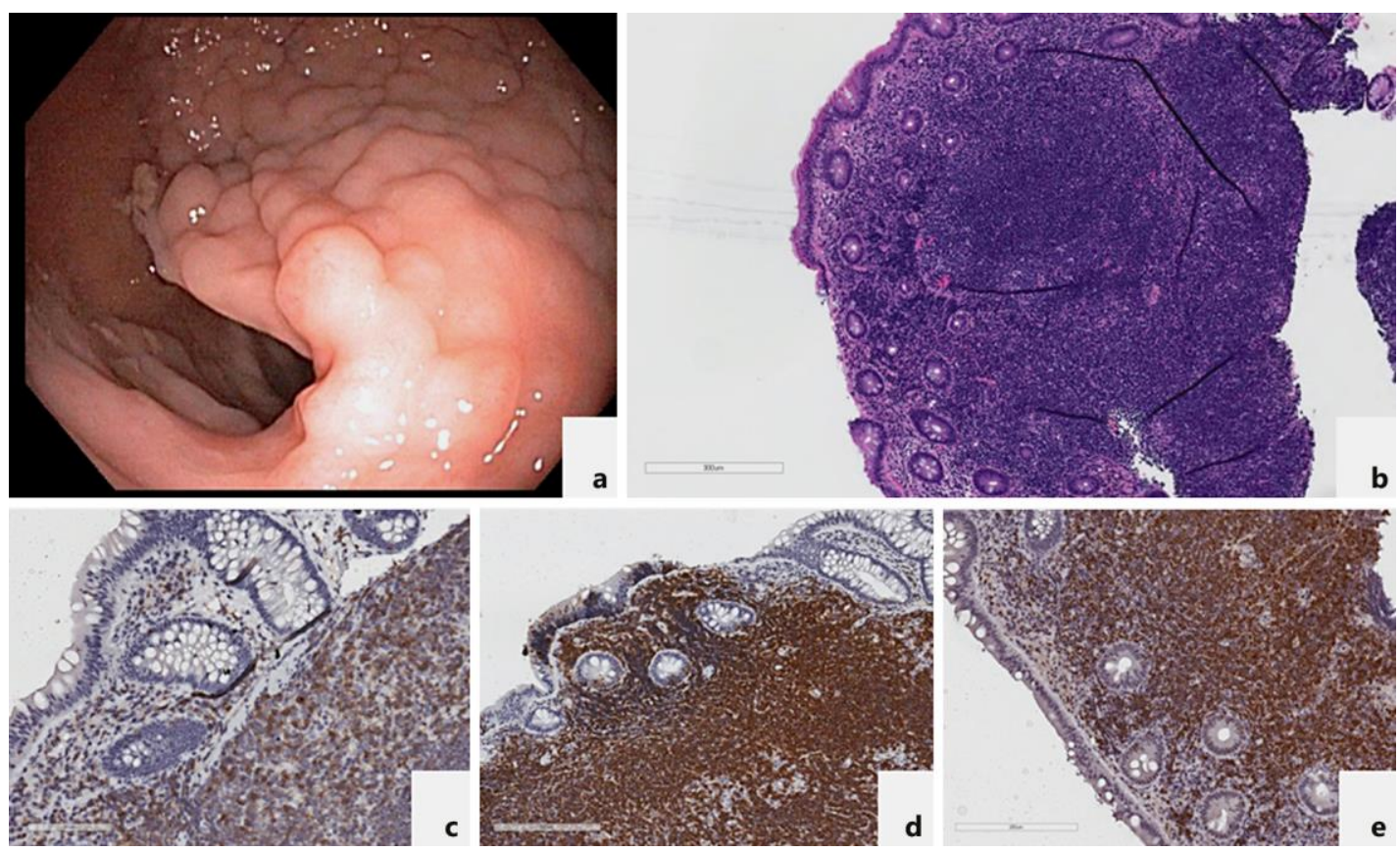

Fig. 1. Staging colonoscopy findings. a Colonoscopy with multiple colonic polyps. b Dense lymphocytic infiltrate on hematoxylin and eosin staining (scale bar: $300 \mu \mathrm{m}$ ). c CD5 positivity (scale bar: $90 \mu \mathrm{m}$ ). $\mathbf{d}$ CD20 positivity (scale bar: $200 \mu \mathrm{m}$ ). e Cyclin D1 positivity (scale bar: $200 \mu \mathrm{m}$ ). This staining pattern along with CD23 negativity and the classic MCL genetic abnormality of $\mathrm{t}(11 ; 14)(\mathrm{q} 13 ; \mathrm{q} 32)$ are consistent with MCL.

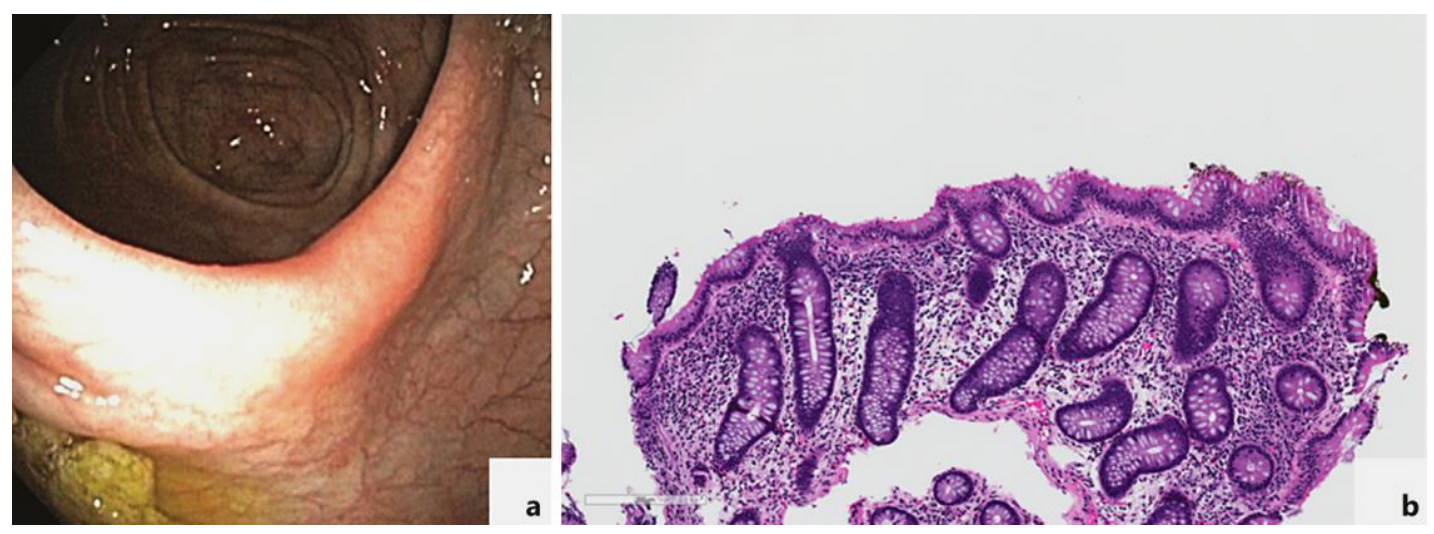

Fig. 2. Repeat colonoscopy 4 months after aggressive immunotherapy, chemotherapy, and autologous bone marrow transplantation, showing normalization of the mucosa. a Normal colon mucosa. $\mathbf{b}$ Hematoxylin and eosin staining without prominent lymphocytic infiltrate (scale bar: $200 \mu \mathrm{m}$ ). 